Correspondence: M. Haschke, Division of Clinical Pharmacology and Toxicology, University Hospital Basel, Petersgraben 4, CH-4031 Basel, Switzerland. E-mail: HaschkeM@uhbs.ch

Statement of Interest: None declared.

\section{REFERENCES}

1 Von Drygalski A, Curtis BR, Bougie DW, et al. Vancomycininduced immune thrombocytopenia. N Engl J Med 2007; 356: 904-910.

2 Documed. Product information dobutamine injection. Arzbeunuttel Kompendium der Schweiz. [Swiss Compendium of Therapeutic Drugs.] www.documed.ch Date last updated: 2005. Date last accessed: December 1, 2009.
3 George JN, Raskob GE, Shah SR, et al. Drug-induced thrombocytopenia: a systematic review of published case reports. Ann Int Med 1998; 129: 886-890.

4 Lee SH, Rubin LJ. Current treatment strategies for pulmonary arterial hypertension. J Intern Med 2005; 258: 199-215.

5 US Food and Drug Administration. Ventavis (iloprost) inhalation solution September 2008. MedWatch FDA Safety Information and Adverse Event Reporting Program. Date last updated: June 19, 2009. Date last accessed: December 1, 2009.

6 Uppsala Monitoring Centre World Health Organization collaborating Centre for International Drug Monitoring. VigiBase ${ }^{\mathrm{TM}}$ Services. www.umc-products.com Date last accessed: December 1, 2009.

7 Chin KM, Channick RN, de Lemos JA, et al. Hemodynamics and epoprostenol use are associated with thrombocytopenia in pulmonary arterial hypertension. Chest 2009; 135: 130-136.

\title{
Azithromycin treatment failure in macrolide-resistant Mycoplasma pneumoniae pneumonia
}

\section{To the Editors:}

Mycoplasma pneumoniae is one of the most common bacterial causes of community-acquired pneumonia (CAP) [1]. Although most cases are mild, life-threatening diseases are also reported [2]. Clinical guidelines have recommended macrolides for treatment of community-acquired infection to target atypical organisms including M. pneumoniae [3] but there have been an increasing number of reports of macrolideresistant $M$. pneumoniae isolated from clinical specimens [4]. Although there are studies suggesting poorer responses to macrolide in patients infected with macrolide-resistant strains, these are mainly limited to patients with mild disease. Here, we report a case of CAP due to macrolide-resistant M. pneumoniae with poor response to azithromycin but rapid resolution after tigecycline.

In October 2009, a 24-yr-old female was admitted to our hospital with a 4-day history of fever, sore throat, cough and myalgia, associated with nausea, vomitting and diarrhoea. 2 months before admission, she was hospitalised for acute pyelonephritis due to Escherichia coli. Otherwise, her past health was unremarkable. She traveled to Xi'an, China, for 7 days and returned to Hong Kong 10 days before the onset of symptoms. While in Xi'an, she stayed with her father, who had an episode of suspected respiratory tract infection, presenting with cough and sputum.

2 days before admission, she attended the accident and emergency department of a regional hospital in Hong Kong SAR (China). A chest radiograph showed right middle zone consolidation (fig. 1a). Her temperature was $39.4^{\circ} \mathrm{C}$, blood pressure was $101 / 57 \mathrm{mmHg}$, pulse was 111 beats $\cdot \mathrm{min}^{-1}$, respiratory rate was 16 breaths $\cdot \mathrm{min}^{-1}$ and oxygen saturation was $98 \%$ while breathing ambient air. She was discharged with amoxicillin-clavulanate (1,000 $\mathrm{mg}$ twice daily) and paracetamol. Because of persistent symptoms 1 day before admission, azithromycin (500 mg once daily) and oseltamivir (75 mg twice daily) were added as treatment for CAP. RT-PCR of nasopharyngeal aspirate for influenza, collected 1 day before
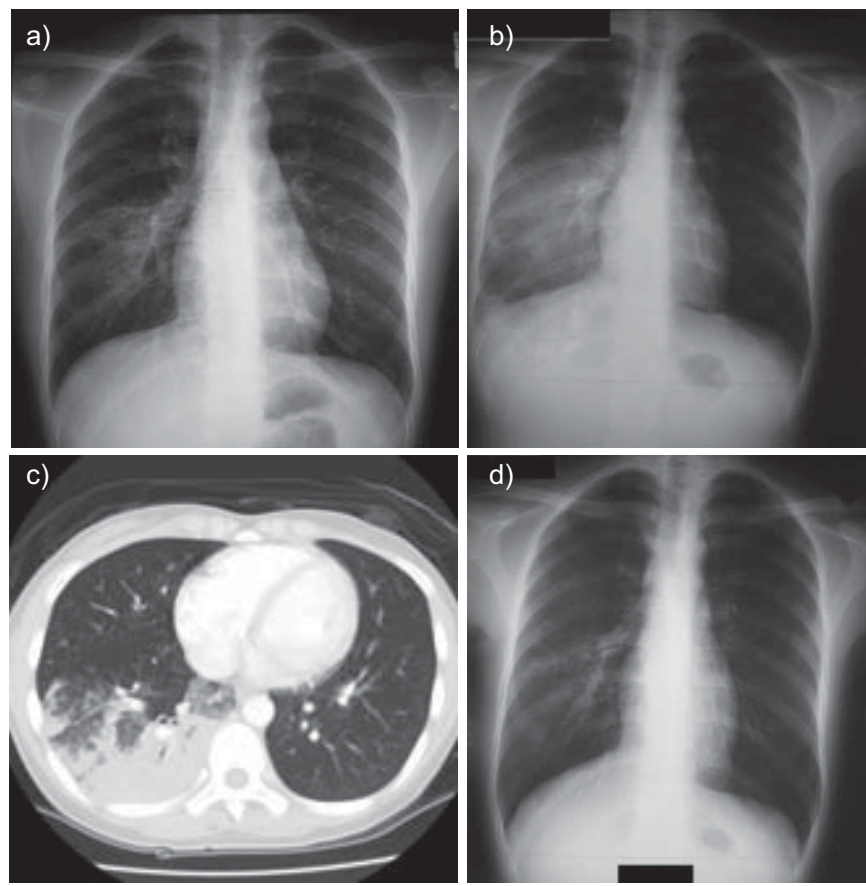

FIGURE 1. Radiographical findings. a) Chest radiograph taken 2 days before admission, showing right middle zone consolidation. b) Chest radiograph on 7th day of hospitalisation, showing progression of consolidation. c) Computed tomography on 7th day of hospitalisation, showing right lower lobe consolidation with ground-glass opacification. d) Chest radiograph on 17th day of hospitalisation, showing resolution of consolidation. 
admission, was negative. Due to progression of consolidation at the right middle and lower zone, she was admitted.

After admission, there was persistent fever and further progression of lung infiltration on chest radiograph (fig. 1b), despite being given: oral azithromycin, $500 \mathrm{mg}$ once daily for 9 days (day -1-8 of hospitalisation); intravenous ceftriaxone, $1 \mathrm{~g}$ once daily (day 2-4 hospitalisation); and piperacillintazobactam, $4.5 \mathrm{~g}$ 8-hourly (day 4-8 hospitalisation). Gram stains of sputum on admission (after 2 days of amoxicillinclavulanate and azithromycin) revealed three or more white blood cells, one or more epithelial cells, and culture grew Pseudomonas aeruginosa. Ziehl-Neelsen staining for acid-fast bacilli, urine for Legionella antigen, nasopharyngeal aspirate immunofluorescence for influenza A and B, parainfluenza virus $1-3$, adenovirus and respiratory syncytial virus, and RTPCR for pandemic influenza H1N1 were all negative. Two sets of blood cultures were negative after 7 days of incubation. Computed tomography with contrast of the thorax was performed on day 7 of hospitalisation, which revealed right lower lobe consolidation with ground-glass opacification and small, scattered ground-glass opacities at posterior right upper lobe (fig. 1c).

Due to persistent fever and pneumonia, antibiotics were switched to intravenous tigecycline (100 mg for first dose, then $50 \mathrm{mg}$ 12-hourly on day 10 of hospitalisation). There was rapid resolution of fever and improvement in lung infiltration (fig. 1d). C-reactive protein decreased from a maximum of $82.5 \mathrm{mg} \cdot \mathrm{L}^{-1}$ (1 day before starting tigecycline) to $16.3 \mathrm{mg} \cdot \mathrm{L}^{-1}$ (2 days after starting tigecycline). The patient was discharged on day 19 of hospitalisation.

Bronchoscopy was performed on day 10 of hospitalisation. Bronchoalveolar lavage (BAL) gave mixed growth of commensals in culture. Work-up for tuberculosis, including ZiehlNeelsen staining and PCR for tuberculosis, were all negative. PCR for M. pneumoniae of the BAL (collected on day 10) and nasopharyngeal aspirate (collected on day 2) were positive, but negative for Chlamydophila. The bacterial DNA load in the two samples was assessed semiquantitatively by serial 10-fold dilutions of the DNA extracts. The BAL and nasopharyngeal aspirate samples remained PCR-positive up to the $10^{-3}$ and $10^{-2}$ dilutions, respectively, suggesting that the bacterial DNA load in the lower respiratory tract was higher than the upper respiratory tract. PCR for M. pneumoniae of the serum, collected on days 5 and 12 of hospitalisation, was negative. Paired serology, collected 10 days apart (on days 2 and 12), showed a rise of antibody titre against $M$. pneumoniae from $<1: 40$ to $1: 10,240$ by particle agglutination assay, but there was no rise in antibody titre for Chlamydia group, Coxiella burnetii, influenza virus type A and B, adenovirus, parainfluenza virus type 1, 2 and 3, or respiratory syncytial virus.

Because of treatment failure, despite 9 days of azithromycin therapy, sequencing of the 23S ribosomal (r)RNA was performed. A-to-G transition at nucleotide 2,063 was found in both the nasopharyngeal aspirate and BAL, collected on days 2 and 10 of hospitalisation, respectively.

We have reported here the investigation of a case of M. pneumoniae pneumonia that failed to respond to prolonged azithromycin treatment. The strain was found to possess a mutation in the 23S rRNA associated with in vitro resistance to azithromycin and other macrolides. The finding is important because there has been a recent surge of macrolide resistance among clinical isolates of $M$. pneumoniae in some parts of the world. This rapid increase was first reported in Japan, where the incidence of macrolide-resistant strains isolated from children with M. pneumoniae pneumonia increased from $0 \%$ in 2002 to $>30 \%$ in 2006, with a minimum inhibitory concentration (MIC) for erythromycin, clarithromycin and azithromycin, determined by microdilution methods of $\geqslant 16 \mu \mathrm{g} \cdot \mathrm{mL}^{-1}$ [4]. In a study involving Chinese children, macrolide resistance was detected in 46 out of 50 M. pneumoniae strains [5]. Resistance was most commonly attributed to a mutation in the domain $\mathrm{V}$ of the $23 \mathrm{~S}$ rRNA. In a report from China, it was found that all macrolideresistant strains of $M$. pneumoniae harbour an A-to-G transition mutation at position 2,063 in the 23S rRNA gene [6]. The MIC of strains with the A2063G mutation is slightly lower for azithromycin $\left(\mathrm{MIC} 90=64 \mu \mathrm{g} \cdot \mathrm{mL}^{-1}\right)$ than clarithromycin or erythromycin (MIC90 $>128 \mu \mathrm{g} \cdot \mathrm{mL}^{-1}$ ). In Japan, other mutations in the 23S rRNA gene that resulted in high-level resistance (MIC $\geqslant 256 \mu \mathrm{g} \cdot \mathrm{mL}^{-1}$ for erythromycin and clarithromycin and $16 \mu \mathrm{g} \cdot \mathrm{mL}^{-1}$ for azithromycin) include A-to-C transversion at position 2,063 and A-to-G transition at position 2,064 [7]. A C-to-G transversion at position 2,617 was also found, but this mutation was associated with low-level macrolide resistance (MIC for erythromycin, clarithromycin and azithromycin was 8 , 1 and $0.031 \mu \mathrm{g} \cdot \mathrm{mL}^{-1}$ respectively).

However, in vitro resistance does not necessarily equate to clinical failure, as demonstrated by azithromycin resistance in Streptococcus pneumoniae infection. Despite numerous reports of detection of macrolide-resistant M. pneumoniae from clinical specimens, there have been few studies on the clinical impact of resistant strains. Children infected with macrolide-resistant M. pneumoniae have a longer duration of fever after initiating macrolide therapy than those infected with a macrolidesensitive strain, and macrolide was more likely to change to another antimicrobial [8]. However, in the study by SUZUKI et al. [8], patients infected with macrolide-resistant strains were more likely to receive erythromycin or clarithromycin than azithromycin. No cohort studies have been performed in an adult population. A previous case report described a 28 -yr-old lady with $\mathrm{CAP}$, who had persistent symptoms after clarithromycin therapy, and later responded to intravenous pazufloxacin [9]. The strain was found to carry the A-to-G transition at position 2,063. For our patient, despite 9 days of azithromycin therapy, there was persistent fever and radiological progression of consolidation. However, after initiation of tigecycline, fever subsided within $24 \mathrm{~h}$, and there was rapid resolution of pulmonary infiltrates. As the patient travelled to mainland China shortly before the onset of symptoms, where she has had contact with her father with respiratory symptoms, it is possible that she could have acquired the resistant organism from Xi'an. There has not been any published data on macrolide-resistant M. pneumoniae in this city, but a high prevalence of macrolide resistance has been found in Shanghai, China [5].

The optimal treatment for a serious infection caused by macrolide-resistant $M$. pneumoniae remains uncertain. Pharmacokinetics and in vitro data indicate that tetracyclines and fluoquinolones are potential options. For macrolideresistant strains with the A2063G mutation, the MIC was 
$0.0625-1 \mu \mathrm{g} \cdot \mathrm{mL}^{-1}$ for minocycline and $0.0625-0.125 \mu \mathrm{g} \cdot \mathrm{mL}^{-1}$ for moxifloxacin [4]. Tigecycline, a glycylcyline, has a more favourable pharmacokinetic and pharmacodynamic profile than the tetracyclines. The MIC of tigecycline (ranging 0.125 $0.5 \mu \mathrm{g} \cdot \mathrm{mL}^{-1}$ ) against $M$. pneumoniae is lower than that of tetracycline $\left(0.5-4 \mu \mathrm{g} \cdot \mathrm{mL}^{-1}\right)$ and is similar to minocycline $\left(0.06-0.5 \mu \mathrm{g} \cdot \mathrm{mL}^{-1}\right)$ [10]. However, further clinical trials would be required to determine whether this translates to clinical superiority. Since both tetracyclines and fluoroquinolones are contraindicated in children, management of serious infection caused by macrolide-resistant $M$. pneumoniae would be challenging.

In this report, we have provided evidence that a transition from A-to-G at position 2,063 position to be associated with treatment failure. We propose that other antibiotics active against M. pneumoniae, such as tetracyclines or fluoroquinolones, should be used if a patient has clinical failure after macrolide therapy. Local surveillance would be important to determine the prevalence of macrolide resistance among M. pneumoniae, and will provide important information regarding empirical therapy against $M$. pneumoniae.

\section{K.K.W. To, K-H. Chan, Y-F. Fung, K-Y. Yuen and P-L. Ho}

Division of Infectious Diseases, Dept of Microbiology and Carol Yu Centre for Infection, The University of Hong Kong, Hong Kong SAR, China.

Correspondence: P-L. Ho, Division of Infectious Diseases, Dept of Microbiology and Carol Yu Centre for Infection, The University of Hong Kong, Queen Mary Hospital, Pokfulam, Hong Kong SAR, China. E-mail: plho@hkucc.hku.hk

Support Statement: The work is supported by research grants from the Research Fund for the Control of Infectious Diseases (RFCID) of the Health, Welfare and Food Bureau of the Hong Kong SAR Government.

Statement of Interest: None declared.
Acknowledgements: We would like to thank A. Wong (Public Health Laboratory Centre of Hong Kong, Hong Kong SAR, China) for assistance with the laboratory investigation.

\section{REFERENCES}

1 Johansson N, Kalin M, Tiveljung-Lindell A, et al. Etiology of community-acquired pneumonia: increased microbiological yield with new diagnostic methods. Clin Infect Dis 2010; 50: 202-209.

2 Miyashita N, Obase Y, Ouchi K, et al. Clinical features of severe Mycoplasma pneumoniae pneumonia in adults admitted to an intensive care unit. I Med Microbiol 2007; 56: 1625-1629.

3 Lim WS, Baudouin SV, George RC, et al. BTS guidelines for the management of community acquired pneumonia in adults: update 2009. Thorax 2009; 64: Suppl. 3, iii1-iii55.

4 Morozumi M, Takahashi T, Ubukata K. Macrolide-resistant Mycoplasma pneumoniae: characteristics of isolates and clinical aspects of community-acquired pneumonia. J Infect Chemother 2010; 16: 78-86.

5 Xin D, Mi Z, Han X, et al. Molecular mechanisms of macrolide resistance in clinical isolates of Mycoplasma pneumoniae from China. Antimicrob Agents Chemother 2009; 53: 2158-2159.

6 Liu Y, Ye X, Zhang H, et al. Antimicrobial susceptibility of Mycoplasma pneumoniae isolates and molecular analysis of macrolide-resistant strains from Shanghai, China. Antimicrob Agents Chemother 2009; 53: 2160-2162.

7 Matsuoka M, Narita M, Okazaki N, et al. Characterization and molecular analysis of macrolide-resistant Mycoplasma pneumoniae clinical isolates obtained in Japan. Antimicrob Agents Chemother 2004; 48: 4624-4630.

8 Suzuki S, Yamazaki T, Narita M, et al. Clinical evaluation of macrolide-resistant Mycoplasma pneumoniae. Antimicrob Agents Chemother 2006; 50: 709-712.

9 Isozumi R, Yoshimine $\mathrm{H}$, Morozumi M, et al. Adult communityacquired pneumonia caused by macrolide resistant Mycoplasma pneumoniae. Respirology 2009; 14: 1206-1208.

10 Kenny GE, Cartwright FD. Susceptibilities of Mycoplasma hominis, M. pneumoniae, and Ureaplasma urealyticum to GAR-936, dalfopristin, dirithromycin, evernimicin, gatifloxacin, linezolid, moxifloxacin, quinupristin-dalfopristin, and telithromycin compared to their susceptibilities to reference macrolides, tetracyclines, and quinolones. Antimicrob Agents Chemother 2001; 45: 2604-2608.

DOI: $10.1183 / 09031936.00041910$ 